\title{
Influence of uric acid on the correlation between waist circumference and triglyceride glucose index: an analysis from CHARLS
}

\author{
Jia Zheng ${ }^{1 *}\left(\mathbb{D}\right.$, Min Jiang $^{2}$ and Yanxia Xie ${ }^{3}$
}

\begin{abstract}
Background: Waist circumference (WC) and uric acid (UA) are significantly related. Still, their temporal sequence and how the sequence works on future risk of triglyceride glucose (TyG) are unknown, especially in the Chinese population.

Methods: Cross-lagged panel model was used to analyze the reciprocal, longitudinal relationships among a set of interrelated variables. The mediation model was constructed to test the effect of the relationship between WC and UA on TyG.

Results: A total of 5727 subjects were enrolled in our study population, of which 53.5\% were women, and the mean age was 59.0 (standard deviation, 8.62) years. After adjusting for traditional confounding factors, the results showed that a higher level of baseline WC was significantly associated with a higher level of follow-up UA $(\beta=0.003, P=0.031)$ and follow-up TyG $(\beta=0.003, P<0.001)$; Simultaneously, there was no statistical association between the level of baseline UA and the level of follow-up WC $(\beta=-0.009, P=0.951)$. The mediation effects of UA on WC-TyG were estimated to be $18.1 \%$ in adults, and $36.2 \%$ in women.

Conclusions: The current study demonstrated that higher baseline level of WC probably preceded UA' level in general population. In addition, UA mediated the relationship of WC to TyG, especially in females. And the possible mechanism would require further clarification.
\end{abstract}

Keywords: Waist circumference, Uric acid, Triglyceride glucose, Temporal relationship

\section{Background}

Insulin resistance (IR) [1-5] and uric acid (UA) [6-11] are closely associated with some noncommunicable diseases (NCDs), including cardiovascular disease (CVD), metabolic syndrome (MS), type 2 diabetes mellitus (T2DM), all-Cause and cause-specific mortality. Therefore, early detection and control of IR and UA are necessary to reduce

\footnotetext{
* Correspondence: 1637643374@qq.com

'Department of Clinical Epidemiology, the Fourth Affiliated Hospital of China Medical University, Shenyang 110032, P. R. China

Full list of author information is available at the end of the article
}

the incidence risk of NCDs, especially among the highrisk, symptom free populations.

Recent researches have reported that visceral fat (determined by waist circumference [WC]) is related to IR [1214], and some studies have found that WC is a simpler and more effective indicator used to discriminate IR than other indicators, such as waist to height ratio [15]. Growing evidence demonstrates that abdominal adiposity, evaluated by $\mathrm{WC}$, causes more health risks than total adiposity, evaluated by body mass index (BMI) [16]. In addition, some researches have indicated that the

(c) The Author(s). 2021 Open Access This article is licensed under a Creative Commons Attribution 4.0 International License, which permits use, sharing, adaptation, distribution and reproduction in any medium or format, as long as you give appropriate credit to the original author(s) and the source, provide a link to the Creative Commons licence, and indicate if changes were made. The images or other third party material in this article are included in the article's Creative Commons licence, unless indicated otherwise in a credit line to the material. If material is not included in the article's Creative Commons licence and your intended use is not permitted by statutory regulation or exceeds the permitted use, you will need to obtain permission directly from the copyright holder. To view a copy of this licence, visit http://creativecommons.org/licenses/by/4.0/. The Creative Commons Public Domain Dedication waiver (http://creativecommons.org/publicdomain/zero/1.0/) applies to the data made available in this article, unless otherwise stated in a credit line to the data. 
measurement of obesity was positively correlated to serum uric acid (UA) [17-19]. And on one Mendelian Randomization study relating UA as an early metabolic disorder having upstream effect in development of more traditional risk factors and CVD itself [20]. Some studies have found that increased of serum UA is related to the increased risk of IR-based clinical diseases and the relationship related to gender $[8,21,22]$. The level of serum $\mathrm{UA}$ is also related with the increase of body fat deposition $[23,24]$.

TyG levels, which are associated with IR, play significant roles in predicting the incidence of diabetes and CVD. However, it is unclear whether WC is related to TyG and to what possible extent. To date, there have been few general population studies, particularly prospective cohort studies of Chinese adults who have increased WC and TyG. At the same time, a large American population study showed that the inverse association between serum antioxidant levels and inflammatory markers is moderated by adiposity [25]. On this basic, the possible association of WC and TyG mediated by UA was hypothesized in the current study. Therefore, the current research aimed to explore the relationship between $\mathrm{WC}$ and $\mathrm{UA}$ to TyG level. In addition, a separate subgroup analysis was conducted to evaluate the differential effects of WC, UA, and TyG between male and female.

\section{Methods}

\section{Study participants}

China Health and Retirement Longitudinal Study (CHAR

LS) was a representative survey research nationwide conducted by the National School of Development of Peking University. Between June 2011 and March 2012 (baseline survey), a multistage process with random cluster sampling was conducted to select a representative sample of individuals older than 45 years in 10,287 households in 450 villages/cities. 17,708 individuals were enrolled in the baseline survey through face-to-face household interviews. After the initial recruitment, all 17,708 individuals have been re-surveyed every two years using the same questionnaire as at the baseline. Blood samples were gathered in the year of 2011 and 2015. The detailed information regarding the CHARLS has been described on the website of CHARLS: http://charls.pku.edu.cn/en. The study protocol has been approved by ethical committees of Peking University (IRB 00001052-11,014), and all individuals have provided written informed consent.

This study was a post hoc analysis of CHARLS from 2011 to 2015. Of the 17,708 individuals at baseline, 1099 subjects were younger than 45 years. Participants with detailed follow-up information, major study variables and confounding variables at baseline and follow-up period were included in the study. Participants who had cancer at baseline were excluded. Finally, 5727 subjects were included in our study. The flowchart in Fig. 1 was used to show the detailed information about the sample size of the subjects and the exclusion criteria in the current research.

\section{Exposures and covariates}

Information on participants demographics (gender, age, ethnicity, and education), lifestyle factors (smoking status and drinking status), and history of diseases (diabetes, chronic kidney disease, and hypertension) were collected during the structured household survey. Data on healthy behaviors were obtained from the subjects' self-reported questionnaire, including smoking status (former, current smoker or never), frequency of alcohol consumption (more than once a month, once a month or never). Data on diabetes, chronic kidney disease, and hypertension were collected by trained health staff members.

Data on collection and measurement of cholesterol indexes, fasting plasma glucose, biochemical blood indexes, and other blood pressure indexes are detailed on the website of CHARLS: http://charls.pku.edu.cn/en. The mean of 3 blood pressure values was calculated and used for our analysis. The BMI was calculated using the weight and height indicators; the formula as follows: $\mathrm{BMI}=$ weight $(\mathrm{Kg}) /$ height $^{2}\left(\mathrm{~m}^{2}\right)$.

\section{Dependent variable $(\mathrm{Y})$}

The TyG index was calculated according to the following formula: $\mathrm{TyG}=\ln$ [fasting TG $(\mathrm{mmol} / \mathrm{L}) \times \mathrm{FPG}(\mathrm{mmol} /$ L) $\times 0.5 \times 159.37$ ]. TG and FPG were measured by enzymatic colorimetric test method.

\section{Independent variable $(\mathrm{X})$}

A soft tape was inserted at the navel level to measure WC in a standing pose with a cloth measuring tape. At the same time, all participants needed to do a regular breathing exercise, holding the breath at the end of exhaling and letting the tape out slightly.

\section{Mediators (M)}

UA plus method was used to measure UA.

\section{Statistical analysis}

All analyses were conducted by IBM SPSS version 22.0 and SPSS Amos 22.0 with $P \leq 0.05$ (two-sided) considered statistically significant differences.

Percentiles were calculated used to describe the categorical variables, while arithmetic mean with standard deviation (SD) was calculated for the description of continuous variables that met the normal distribution. The median with interquartile range was used to describe the continuous variables that didn't meet the normal distribution. At the same time, the Student $t$ test, the Mann- 


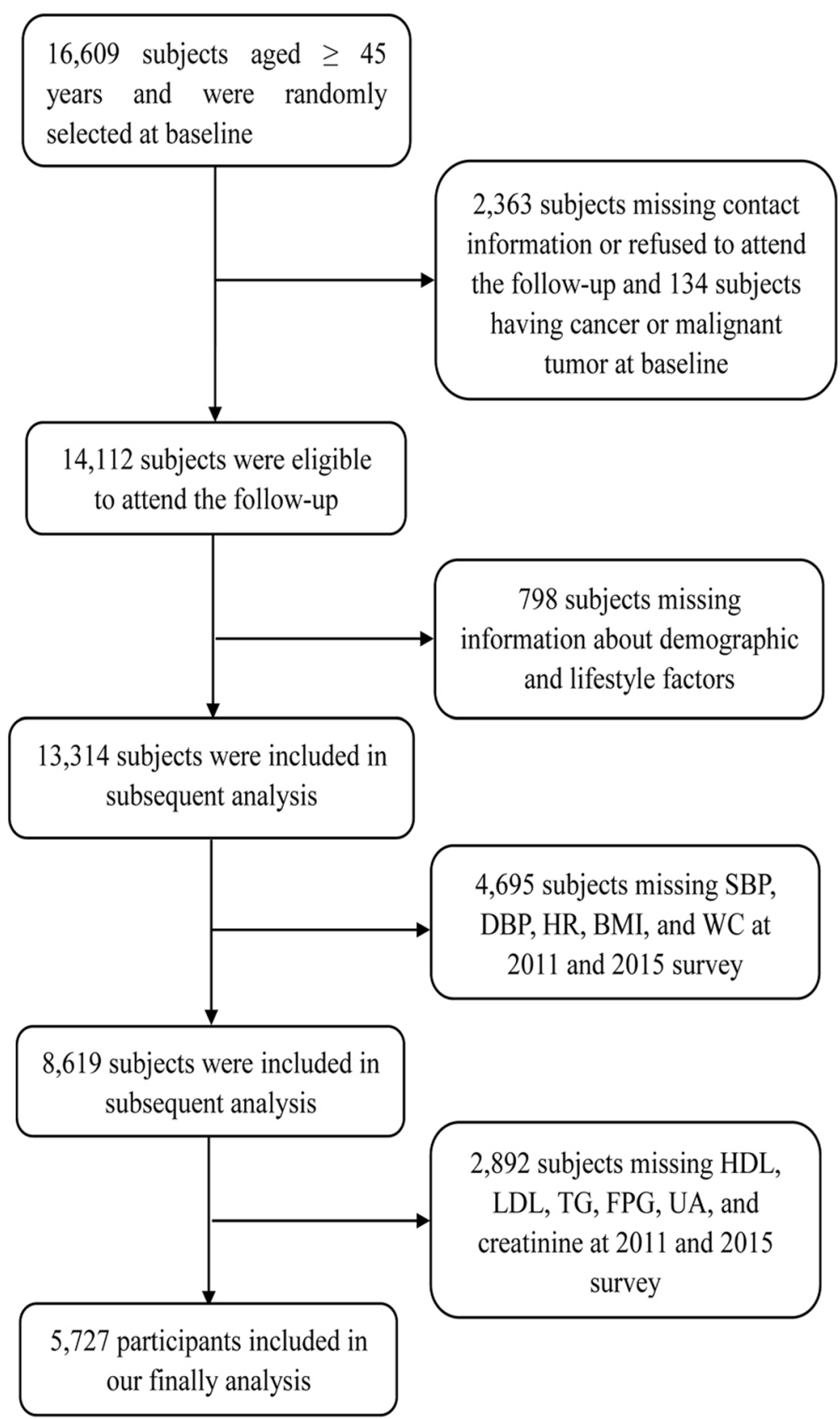

Fig. 1 Flowchart of participants included in this study after inclusion and exclusion

Whitney U test or the Pearson's $\chi^{2}$ test was used for the comparison between male and female.

The linear regression model was constructed to explore whether WC (UA) could predict the future variation of TyG. Future TyG variation and baseline WC (UA) were the dependent and independent variables of the model, respectively. Firstly, the multicollinearity problem among independent variables was examined by the variance expansion factor (VIF). VIF greater than 10 was deemed significant multicollinearity. Secondly, three models were constructed to assess the relationship between baseline (examination at 2011) WC (UA) and future (follow-up at 2015) TyG as follows: model 1: adjusted for baseline WC (UA); model 2: model 1 plus sex, ethnicity and age; model 3: model 2 plus drinking, current smoking, education, BMI, LDL-C, HDL-C, HR, DBP, SBP, and creatinine.

Longitudinal changes of $\mathrm{WC}$ and UA indexes were measured at two follow-up periods. Previous study introduced the theory and application of cross-lagged panel 
design [26]. Overall, the cross-lagged panel model was performed for the longitudinal relationships among different interrelated variables [27]. In Fig. 2, the path with $\beta_{1}$ showed the impact of baseline UA on follow-up WC, and $\beta_{2}$ showed the effect of baseline $\mathrm{WC}$ on follow-up UA. WC and UA values were adjusted in linear regression analysis by baseline and follow-up variables: sex, age, ethnicity, alcohol consumption, current smoking, education, BMI, LDL-C, HDL-C, HR, DBP, SBP, and creatinine. Residuals were saved and after that, Ztransformation was used to standardize the saved residuals (mean $=0 ; \mathrm{SD}=1)$. Root-mean-square residual (RMR) and comparative Fit Index (CFI) and were enrolled for the model fits. RMR $<0.05$ and CFI $>90$ meant a relatively good model fit in the cross-lagged path model. In addition, this analysis model was constructed in groups of men and women, separately.

When the temporal relationship was establised between WC and UA, the mediation model would be fitted to explore the potential influence of the association between UA and WC on TyG. The values of TyG were analyzed by linear regression residual model and standardized by Z-transformation (with mean of 0 and $\mathrm{SD}$ of 1). According to the results of the cross-lagged path analysis model, $\mathrm{X}$ and $\mathrm{M}$ were determined as predictor and mediator, respectively. The detailed mediation model was showed in Fig. 3, which included three models, given by:

$$
\begin{aligned}
& \text { Model } \mathrm{Y}=\beta_{\mathrm{Tol}} \mathrm{X}\left(\beta_{\mathrm{Tol}}=\text { total effect }\right), \\
& \text { Model } \mathrm{M}=\beta_{1} \mathrm{X}\left(\beta_{1}=\text { indirect effect } 1\right),
\end{aligned}
$$

$$
\begin{aligned}
\text { Model } Y=\beta_{2} \mathrm{M}+\beta_{\text {Dir }} \mathrm{X}\left(\beta_{2}\right. & =\text { indirect effect } 2, \beta_{\text {Dir }} \\
& =\text { direct effect }) .
\end{aligned}
$$

The following formula was used to calculate the proportion of the mediation effect, as follows: Mediation effect $(\%)=\frac{\beta_{1} \times \beta_{2}}{\beta_{\text {Tol }}} \times 100 \%$.

\section{Results}

In the end, the study enrolled 5727 participants, of which $53.5 \%$ was female. The mean age of this population was $59.0 \pm 8.62$ years. Detailed baseline and follow-up characteristics of the study population are summarized in Table 1. By comparing the difference between sex groups, the results indicated that HR, DBP, SBP, and FPG were not significantly different at baseline and FPG was not significantly different at follow-up.

Table 2 shows the prospective correlation of baseline WC and follow-up UA and TyG. In model 3, the result revealed that a higher value of $\mathrm{WC}$ was related with a higher follow-up UA value $(\beta=0.003, P=0.031)$ and follow-up TyG level $(\beta=0.003, P<0.001)$.

Table 3 showed the prospective relationship of baseline UA with follow-up WC and TyG. The results indicated that a higher baseline UA value was related to a higher follow-up TyG value $(\beta=0.051, P<0.001)$. However, there was no significant association between baseline UA and follow-up WC $(\beta=-0.009, P=0.951)$.

Results of the cross-lagged path analysis for WC and $\mathrm{UA}$ in the general population with age over 45 are shown in Fig. 2. The $\mathrm{WC}_{\text {baseline }} \rightarrow \mathrm{UA}_{\text {follow-up }}$ path

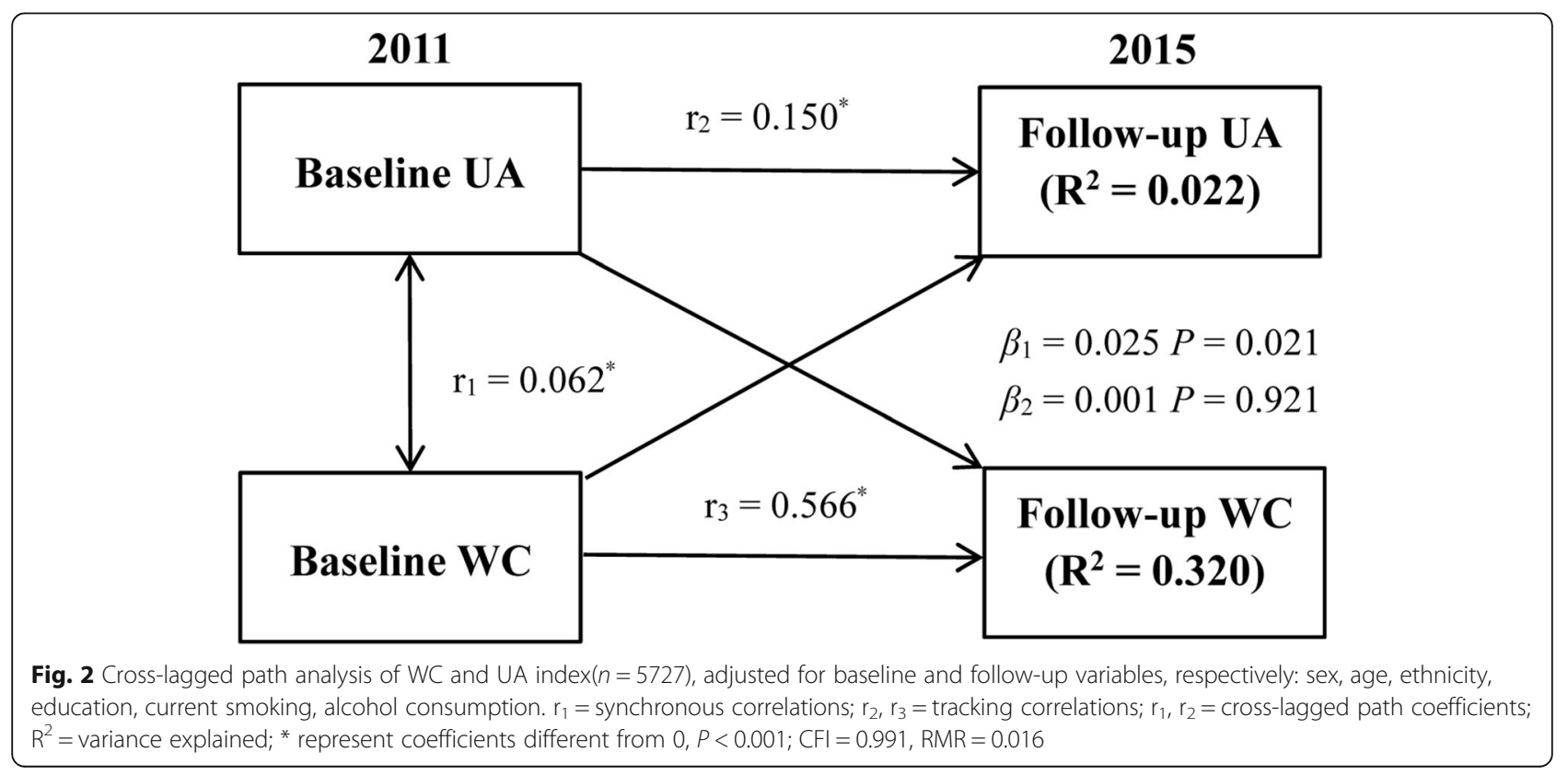




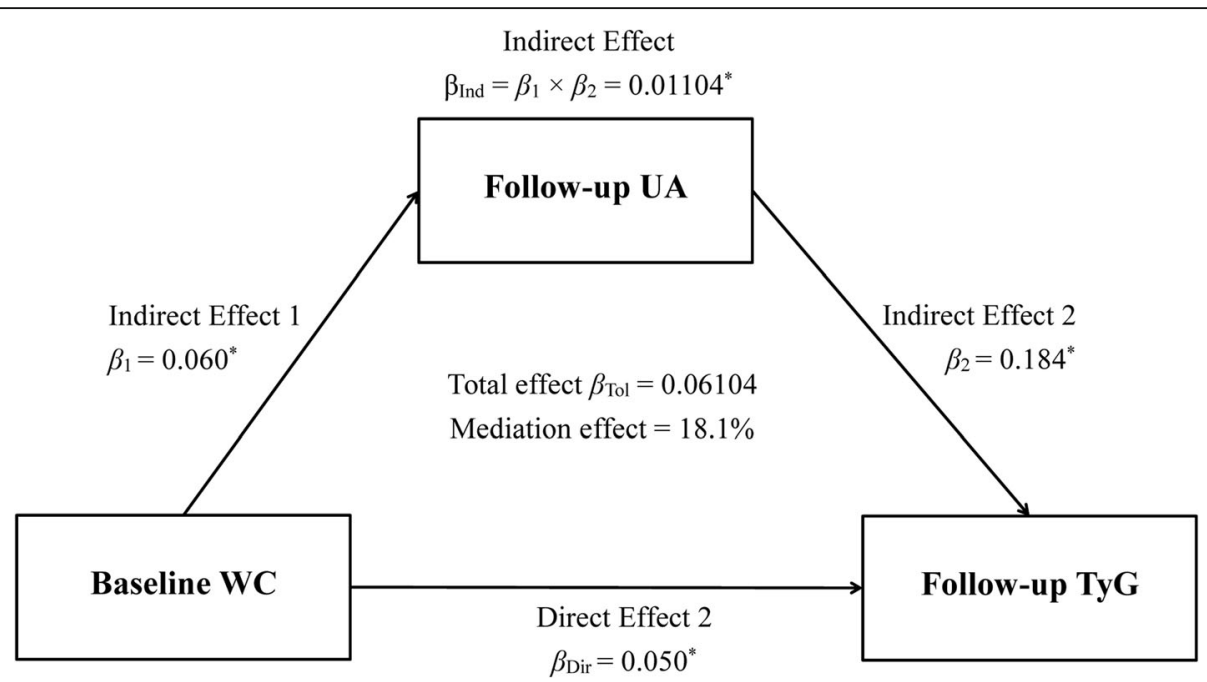

Fig. 3 Mediation effect of UA index on the WC-TyG association $(n=5727),{ }^{*}$ represent coefficients different from $0, P<0.001$

coefficient is $0.025(P=0.021)$. However, the $\mathrm{UA}_{\text {base- }}$ line $\rightarrow \mathrm{WC}_{\text {follow-up }}$ path coefficient is $0.001 \quad(P=0.921)$. The study results showed that a wider WC at baseline resulted in increased UA levels at follow-up, but an increased baseline UA level did not lead to a wider WC. The values of the model fitting parameters in the WC with UA model $(\mathrm{CFI}=0.991$ and $\mathrm{RMR}=0.016)$ showed a relatively good fit to the observed data in our crosslagged path analysis.

Figure 3 shows the mediation effects of follow-up TyG on the relationship between baseline $\mathrm{WC}$ and follow-up UA, adjusted for sex, age, ethnicity, alcohol drinking, current smoking, education, BMI, LDLC, HDL-C, HR, DBP, SBP, and creatinine, after excluding cancer at baseline. The standardized total and direct effect of WC on TyG was 0.06104 and 0.050, respectively. According to the formula in the statistical analysis section, the pooled indirect effect was $0.01104(0.060 \times 0.184)$, while the percentage of the total effects mediated by follow-up UA was $18.1 \%\left(\frac{0.01104}{0.06104} \times 100 \%\right)$.

The results of subgroup analyses between men and women are shown in Figs. 4 and 5. For women, the results showed that the $\mathrm{WC}_{\text {baseline }} \rightarrow \mathrm{UA}_{\text {follow-up }}$ path coefficient is $0.033(P=0.014)$, and the $\mathrm{UA}_{\text {baseline }} \rightarrow \mathrm{WC}_{\text {follow-up }}$ path coefficient is $0.019(P=0.340)$. However, for men, we found that the $\mathrm{WC}_{\text {baseline }} \rightarrow \mathrm{UA}_{\text {follow-up }}$ path coefficient is $0.015(P=0.407)$, and the $\mathrm{UA}_{\text {baseline }} \rightarrow \mathrm{WC}_{\text {follow-up }}$ path coefficient is $-0.013(P=0.444)$. These results indicated that an increase in baseline WC might affect a subsequent UA increase, but only in women, not in men. For women, the percentage of the overall effect mediated by follow-up UA was estimated at $36.2 \%\left(\frac{0.01362}{0.03762} \times 100 \%\right)$.

\section{Discussion}

Results of current research were 4-fold: (1) clarifying the temporal relationship between $\mathrm{WC}$ and $\mathrm{UA}$ ( $\mathrm{WC}_{\text {base- }}$ line $\rightarrow \mathrm{UA}_{\text {follow-up); }}$ (2) verifying UA mediate effect on the relationship between WC and TyG; (3) estimating the percentages of UA mediation effect on WC - TyG (18.1\%); (4) and finding the mediation effect of UA on WC - TyG in women not in men.

It is widely known that with bad dietary habits and sedentary lifestyle, together with the increase of social pressure. Furthermore, the global obesity rate has shown apparent upward trend [1,2]. Studies have shown a strong relationship between obesity and disease, and abnormal fat distribution in the body, especially the accumulation of abdominal fat, has a higher correlation with the occurrence of IR [12-14]. WC is a simple and reliable measure of central obesity. Studies have demonstrated that WC can be used as an indicator to assess early insulin secretion [15]. In addition, serum UA and obesity are related to an elevated level of IR and the incidence of MS [21]. Other studies have demonstrated that UA was an independent risk factor for gout and some major public health threats, such as CVD, MS, and even stroke [6-10]. WC as the obesity indicator is considered the main risk factor for hyperuricemia [23, 24], however, the temporal relationship between $\mathrm{WC}$ and hyperuricemia is still controversial. In this study, the cross-lagged path analysis was applied to explore the chicken-and-egg question because it is a practical statistical approach to analyze the causal relationship between two intercorrelated variables. In current study, the results revealed a unidirectional relationship from WC to UA. In addition, because sex may influence the level of $\mathrm{WC}$, UA, and 
Table 1 Baseline Characteristics of Study Participants by sex and abdominal obesity $(n=5727)$

\begin{tabular}{|c|c|c|c|c|}
\hline Characteristic & Men $(n=2663)$ & Women $(n=3064)$ & $P$ & Total \\
\hline \multicolumn{5}{|l|}{ Baseline (2011) } \\
\hline Age (years) & $59.9(8.57)$ & $58.2(8.58)$ & $<0.001$ & $59.0(8.62)$ \\
\hline \multicolumn{5}{|l|}{ Ethnicity, n (\%) } \\
\hline Han & $2491(93.5)$ & $2820(92.0)$ & 0.029 & $5311(92.7)$ \\
\hline Others & $172(6.5)$ & $244(8.0)$ & & $416(7.3)$ \\
\hline \multicolumn{5}{|l|}{ Education, n (\%) } \\
\hline Illiteracy & $852(32.0)$ & $1853(60.5)$ & $<0.001$ & $2705(47.2)$ \\
\hline Primary school & $764(28.7)$ & $572(18.7)$ & & $1336(23.3)$ \\
\hline Middle school & 709 (26.6) & $456(14.9)$ & & $1165(20.3)$ \\
\hline High school & $222(8.3)$ & $146(4.8)$ & & $368(6.4)$ \\
\hline Tertiary high school or above & $116(4.4)$ & $37(1.2)$ & & $153(2.7)$ \\
\hline Current smoking, n (\%) & $2010(75.5)$ & $240(7.8)$ & $<0.001$ & $2250(39.3)$ \\
\hline Drinking $\geq 1$ times/month, $\mathrm{n}(\%)$ & $1214(45.6)$ & $225(7.3)$ & $<0.001$ & $452(7.9)$ \\
\hline $\mathrm{SBP}(\mathrm{mmHg})$ & $129.7(19.96)$ & $130.0(21.59)$ & 0.537 & $129.8(20.85)$ \\
\hline $\mathrm{DBP}(\mathrm{mmHg})$ & 75.9 (12.34) & $75.4(11.70)$ & 0.121 & $75.6(12.00)$ \\
\hline HR (beats/min) & 71.9 (10.79) & $72.4(9.70)$ & 0.051 & $72.2(10.22)$ \\
\hline $\mathrm{HDL}-\mathrm{C}(\mathrm{mg} / \mathrm{dL})$ & $48.33(39.05-59.54)$ & $50.26(41.37-60.31)$ & $<0.001$ & $49.48(40.21-59.92)$ \\
\hline LDL-C (mg/dL) & $109.41(89.30-131.44)$ & $118.30(96.26-142.66)$ & $<0.001$ & $114.05(93.17-137.24)$ \\
\hline BMI (Kg/m2) & $23.0(3.56)$ & $24.1(3.94)$ & $<0.001$ & $23.6(3.81)$ \\
\hline Waist Circumference (cm) & $84.1(11.83)$ & $84.8(12.81)$ & 0.044 & $84.5(12.37)$ \\
\hline Uric Acid (mg/dL) & $4.88(1.233)$ & $3.98(1.042)$ & $<0.001$ & $4.40(1.221)$ \\
\hline Creatinine (mg/dL) & $0.87(0.189)$ & $0.69(0.136)$ & $<0.001$ & $0.77(0.186)$ \\
\hline FPG (mmol/L) & $6.10(1.931)$ & $6.12(1.925)$ & 0.686 & $6.11(1.928)$ \\
\hline $\mathrm{TG}(\mathrm{mmmol} / \mathrm{L})$ & $1.43(1.147)$ & $1.55(1.025)$ & $<0.001$ & $1.50(1.085)$ \\
\hline TyG* & $6.33(0.669)$ & $6.44(0.643)$ & $<0.001$ & $6.39(0.658)$ \\
\hline \multicolumn{5}{|l|}{ Follow-up (2015) } \\
\hline Current smoking, n (\%) & $1392(52.3)$ & $167(5.5)$ & $<0.001$ & $1559(27.2)$ \\
\hline Drinking $\geq 1$ times/month, $\mathrm{n}(\%)$ & $1187(44.6)$ & $243(7.9)$ & $<0.001$ & $1430(25.0)$ \\
\hline $\mathrm{SBP}(\mathrm{mmHg})$ & $130.5(19.73)$ & $129.4(20.23)$ & 0.032 & $129.9(20.00)$ \\
\hline $\mathrm{DBP}(\mathrm{mmHg})$ & $76.3(11.35)$ & $74.7(10.89)$ & $<0.001$ & $75.4(11.13)$ \\
\hline$H R^{*}$ & $73.3(11.19)$ & $74.3(10.19)$ & 0.001 & $73.8(10.68)$ \\
\hline $\mathrm{HDL}-\mathrm{C}$ & $48.26(41.70-56.70)$ & 51.35 (44.79-58.69) & $<0.001$ & $50.19(43.24-57.92)$ \\
\hline LDL-C & 96.52 (79.54-115.06) & 105.79 (86.49-123.94) & $<0.001$ & $101.54(83.01-120.08)$ \\
\hline BMI (Kg/m2) & $23.2(3.73)$ & $24.3(4.24)$ & $<0.001$ & $23.8(4.05)$ \\
\hline Waist Circumference (cm) & $84.3(13.80)$ & $85.7(13.41)$ & $<0.001$ & $85.0(13.61)$ \\
\hline Uric Acid (mg/dL) & $5.46(1.419)$ & $4.48(1.215)$ & $<0.001$ & $4.94(1.403)$ \\
\hline Creatinine (mg/dL) & $0.92(0.304)$ & $0.72(0.207)$ & $<0.001$ & $0.81(0.275)$ \\
\hline $\mathrm{FPG}(\mathrm{mmol} / \mathrm{L})$ & $5.75(1.997)$ & $5.82(2.022)$ & 0.207 & $5.79(2.010)$ \\
\hline $\mathrm{TG}(\mathrm{mmmol} / \mathrm{L})$ & $1.51(0.996)$ & $1.73(1.044)$ & $<0.001$ & $1.62(1.028)$ \\
\hline TyG & $6.34(0.643)$ & $6.50(0.627)$ & $<0.001$ & $6.43(0.640)$ \\
\hline
\end{tabular}

Abbreviations: UA uric acid, WC waist circumference, TyG triglyceride-glucose, SBP systolic blood pressure, DBP diastolic blood pressure, $H R$ heart rate, BMI body mass index, HDL-C high-density lipoprotein cholesterol, $L D L-C$ low-density lipoprotein cholesterol, TG Triglycerides, $F P G$ fasting blood glucose TyG $=$ In [fasting TG $(\mathrm{mmol} / \mathrm{L}) *$ FPG $(\mathrm{mmol} / \mathrm{L}) * 0.5 * 159.37]$ $\mathrm{BMI}=$ Weight $(\mathrm{kg}) /$ height $(\mathrm{m})^{2}$ 
Table 2 Prospective associations of baseline WC with follow-up UA and TyG

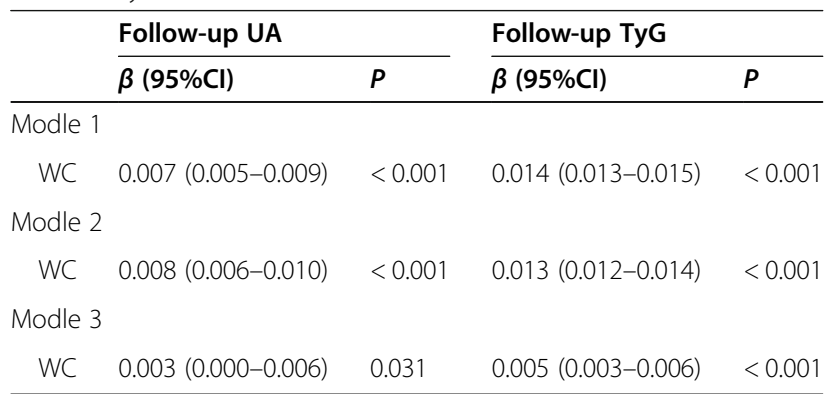

Model 1: adjusted for baseline UA

Model 2: adjusted for factors in model 1 and baseline age, ethnicity and sex

Model 3: adjusted for all variables in model 2 plus baseline education, current smoking, alcohol drinking, SBP, DBP, HR, BMI, HDL-C, LDL-C, creatinine Abbreviations: UA uric acid, WC, waist circumference, TyG triglyceride-glucose, $S B P$ systolic blood pressure, $D B P$ diastolic blood pressure, $H R$ heart rate, $B M I$ body mass index, HDL-C high-density lipoprotein cholesterol, $L D L-C$ lowdensity lipoprotein cholesterol

TyG, a subgroup analysis was conduced in male and female, separately. Unidirectional relationship from WC to UA was significantly stronger in female than that in male. UA mediated the effect of WC on TyG, and the mediation effect of UA was only observed in women, not in men. The reason for gender differences needs indepth studies.

A previous study has shown that half of diabetes cases could be attributed to the increased serum UA level [28]. The increased level of serum UA is known to be related to hypertension, glucose intolerance, and obesity [29]. A previous study demonstrated that the high level of UA predicted the development of hyperinsulinemia [30]. Therefore, the mediation impact of UA on IR should be considered when examining the association of WC and IR. The high level of UA was positively correlated with the expression level of high-sensitivity Creactive protein [31]. Oxidative stress in adipose tissue is a risk factor for IR because in oxidative stress insulin

Table 3 Prospective associations of baseline UA with follow-up WC and TyG

\begin{tabular}{|c|c|c|c|c|}
\hline & Follow-up WC & $P$ & Follow-up TyG & $P$ \\
\hline \multicolumn{5}{|c|}{ Modle 1} \\
\hline UA & $0.221(-0.033-0.474)$ & 0.088 & $0.037(0.023-0.050)$ & $<0.001$ \\
\hline \multicolumn{5}{|c|}{ Modle 2} \\
\hline UA & $0.552(0.279-0.826)$ & $<0.001$ & $0.074(0.060-0.088)$ & $<0.001$ \\
\hline \multicolumn{5}{|c|}{ Modle 3} \\
\hline UA & $-0.009(-0.284-0.267)$ & 0.951 & $0.051(0.037-0.066)$ & $<0.001$ \\
\hline
\end{tabular}

Model 1: adjusted for baseline WC

Model 2: adjusted for factors in model 1 and baseline age, ethnicity and sex Model 3: adjusted for all variables in model 2 plus baseline education, current smoking, alcohol drinking, SBP, DBP, HR, BMI, HDL-C, LDL-C, creatinine Abbreviations: UA uric acid, WC waist circumference, TyG triglyceride-glucose, $S B P$ systolic blood pressure, $D B P$ diastolic blood pressure, $H R$ heart rate, $B M I$ body mass index, HDL-C high-density lipoprotein cholesterol, LDL-C lowdensity lipoprotein cholesterol sensitivity decreases in adipose tissue. Simultaneously, soluble UA might lead to increased generation of reactive oxygen species (ROS) and elevated tissue NADPH oxidase levels. Increasing serum UA can cause IR by reducing the bioavailability of nitric oxide and lead to oxidative stress in mitochondria. In 2014 Viazzi et al. [32] stated that this mechanism happens because of hyperinsulinemia that acts as an excellent marker in measuring IR. In addition, another two researches have demonstrated that the association between UA and CVD outcomes was U-shaped curve [33, 34]. And this may be the potential reason that this mechanism may also exist in the case of low UA concentrations.

The rising IR-related health burden emphasizes the significance and urgency for searching and managing risk factors for IR. Therefore, early detection and control of IR is crucial, and it should be carried out even in the absence of symptoms. Recently, homeostatic model assessment (HOMA-IR) has been commonly used as measurement index. However, the HOMA-IR is timeconsuming and expensive. Some studies have developed and assessed surrogate markers for IR [35]. One of these biomarkers is the TyG, and it has been examined in different populations with stable results, although these studies were carried out in normal-weight adults [36]. Therefore, the TyG was used in the research.

Prevalence of abdominal obesity has increased and has been considered a major health hazard, which is significantly correlated with the risk of mortality, CVD, as well as other diseases. Comparing to generalized obesity, central obesity is more closely linked to IR. WC as a predicted measure of health risk is a convenient and effective index of abdominal obesity. It has a significant, predictable association with intra-abdominal fat area and volume. One cross-sectional study of participants without diabetes demonstrated that the insulin sensitivity index significantly correlated with WC across populations [36]. Previous literature evidence has found that WC is a suitable proxy measure for central obesity [15]. Studies have shown that with the increase of BMI and adipose tissue, the incidence of T2DM gradually increases [16]. However, the ratio of adipose tissue to muscle will affect the BMI, so the situation of human adipose tissue cannot be entirely and objectively reflected. Additionally, increased triglyceride-rich lipoprotein secretion and impaired clearance of these lipoproteins are the potential mechanisms for central obesity and hypertriglyceridemia [37, 38]. After controlling for BMI, WC as a marker of abdominal obesity, has been significantly associated with T2DM risk [39]. In addition, it has been debated that CVD and IR are associated with the increase in visceral adipose tissue, and WC is a better marker of changes in fat distribution comparing to BMI. 
Men

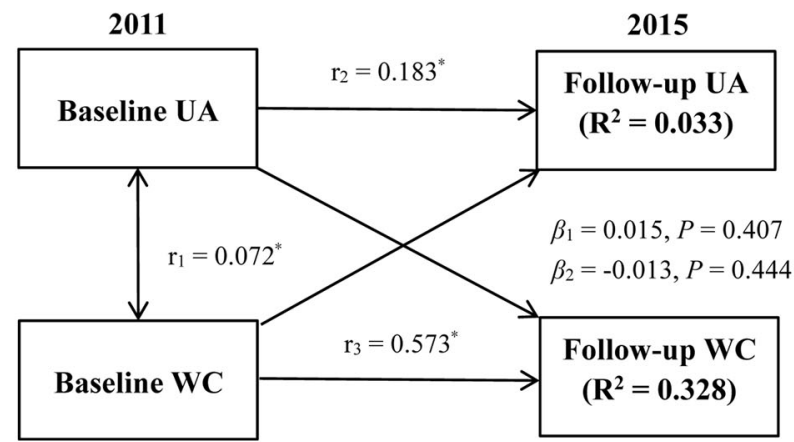

In men: Cross-lagged path analysis of WC and UA index(n=2663), agjusted for baseline and follow-up variables, respectively: sex, age, ethnicity, education, current smoking, alcohol drinking, SBP, DBP, HR, BMI, HDL-C, LDL-C, and creatinine. $\mathrm{r}_{1}=$ synchronous correlations; $\mathrm{r}_{2}, \mathrm{r}_{3}$ $=$ tracking correlations; $\mathrm{r}_{1}, \mathrm{r}_{2}=$ cross-lagged path coefficients; $\mathrm{R}_{2}=$ variance explained); " represent coefficients different from $0, P<0.001$; $\mathrm{CFI}=0.995, \mathrm{RMR}=0.015$
Women

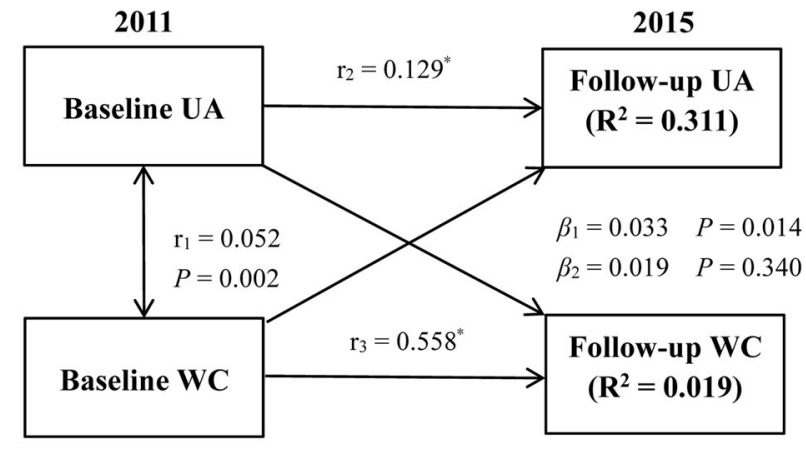

In women: Cross-lagged path analysis of WC and UA index(n=3064), agjusted for baseline and follow-up variables, respectively: sex, age, ethnicity, education, current smoking, alcohol drinking, SBP, DBP, HR, BMI, HDL-C, LDL-C, and creatinine. $\mathrm{r}_{1}=$ synchronous correlations; $\mathrm{r}_{2}, \mathrm{r}_{3}$ $=$ tracking correlations; $\mathrm{r}_{1}, \mathrm{r}_{2}=$ cross-lagged path coefficients; $\mathrm{R}_{2}=$ variance explained); ${ }^{*}$ represent coefficients different from $0, P<0.001$; $\mathrm{CFI}=0.984, \mathrm{RMR}=0.019$

Fig. 4 In men: Cross-lagged path analysis of WC and UA index $(n=2663)$, adjusted for baseline and follow-up variables, respectively: sex, age, ethnicity, education, current smoking, alcohol consumption, SBP, DBP, HR, BMI, HDL-C, LDL-C, and creatinine. $r_{1}=$ synchronous correlations; $r_{2}, r_{3}=$ tracking correlations; $r_{1}, r_{2}=$ cross-lagged path coefficients; $R^{2}=$ variance explained; * ${ }^{*}$ represent coefficients different from $0, P<0.001 ; C F I=0.995, R M R=0.015$; In women: Cross-lagged path analysis of WC and UA index $(n=3064)$, adjusted for baseline and follow-up variables, respectively: sex, age, ethnicity, education, current smoking, alcohol consumption, SBP, DBP, HR, BMI, HDL-C, LDL-C, and creatinine. $r_{1}=$ synchronous correlations; $r_{2}, r_{3}=$ tracking correlations; $r_{1}, r_{2}=$ cross-lagged path coefficients; $R^{2}=$ variance explained; ${ }^{*}$ represent coefficients different from $0, P<0.001 ; C F I=0.984, R M R=0.019$

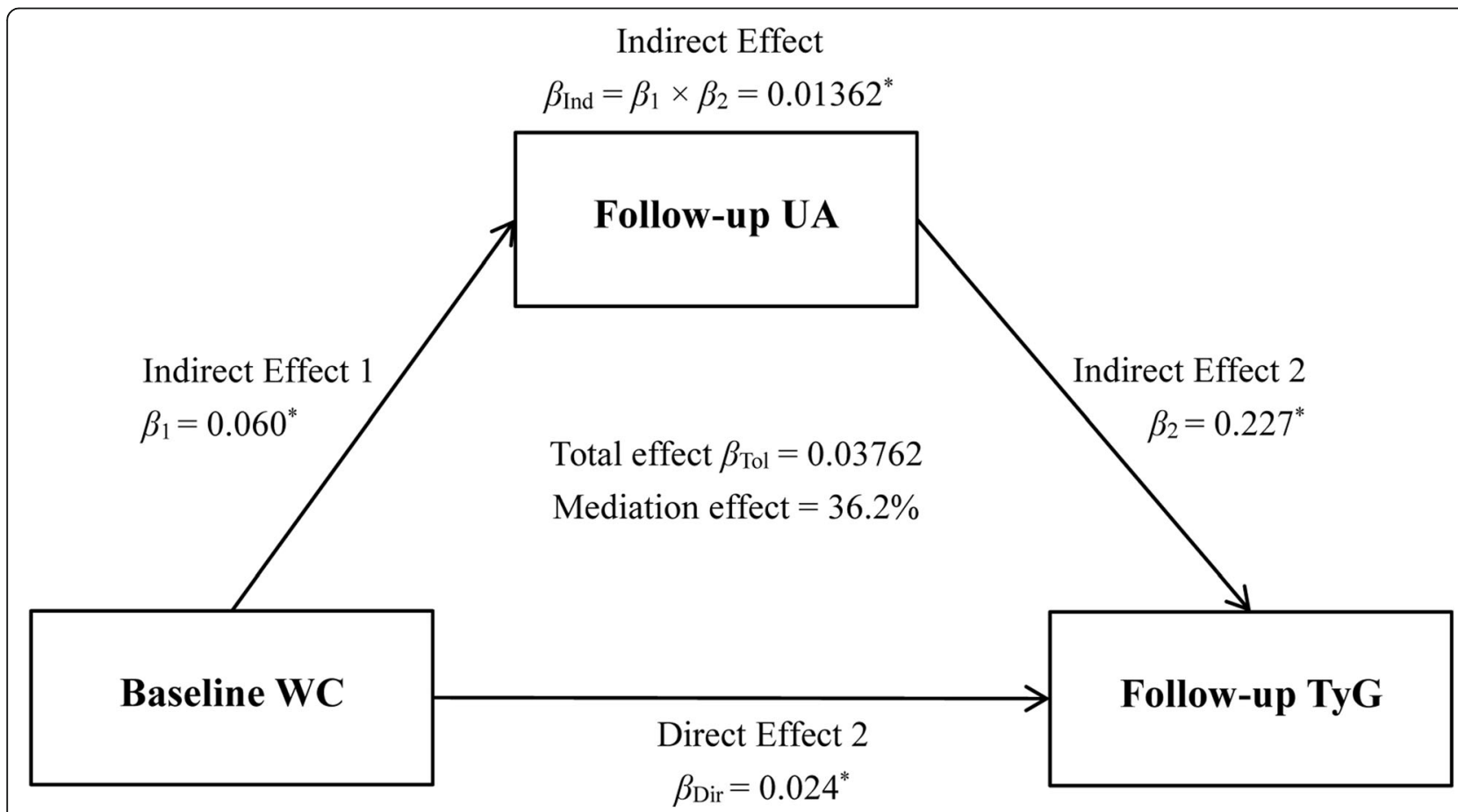

Fig. 5 Mediation effect of UA index on the WC-TyG association $(n=3064)$ in women, * represent coefficients different from $0, P<0.001$ 
The Finnish Diabetes Prevention Study with impaired glucose tolerance risk population showed that the change in UA was significantly related to the change in fasting insulin [40]. The serum level of UA as the purine metabolism, is determined by the level of purine metabolism in the liver, excretion from the kidney, and secretion from the adipose tissue. Some studies proved that central obesity might be associated with hypertriglyceridemia by increasing the triglyceride-rich lipoprotein secretion and impairing this lipoprotein clearance [37, 38]. The mechanism may include the abnormal secretion of very-low-density lipoproteins associated with the increased visceral adiposity [37].

\section{Study strength and limitations}

All WC, UA, and TyG measurements were carried out by trained staff using unified and standardized measuring instrument with high repeatability. Most importantly, we conducted the subgroup analysis between males and females about the UA's mediation effects on WC to IR in a representative sample of the Chinese population with age $\geq 45$ years. The study added to the understanding of the relationship between UA and IR in the context of obesity and metabolic syndrome. Additionally, the current study provided information on alcohol consumption, current smoking, education, BMI, LDL-C, HDL-C, HR, DBP, SBP, and creatinine. After excluding the missing data, only 5727 participants were included in our finally analysis.

\section{Conclusions}

The results of this research demonstrated that the baseline level of WC and UA was associated with the future risk of IR. Furthermore, the temporal relationship between $\mathrm{WC}$ and $\mathrm{UA}$ was $\mathrm{WC}_{\text {baseline }} \rightarrow \mathrm{UA}_{\text {follow-up, and }}$ the mediation effect of UA on WC - TyG was found in women, but not in men. Therefore, early WC detection might be helpful for the primary care physicians to stratify the potential patients that need the further blood screening. Further researches are required to explore the possible underlying mechanism.

\section{Abbreviations}

IR: Insulin resistance; UA: Uric acid; NCDs: Noncommunicable diseases; T2DM: Type 2 diabetes mellitus; MS: Metabolic syndrome;

CVD: Cardiovascular disease; WC: Waist circumference; BMI: Body mass index; CHARLS: China Health and Retirement Longitudinal Study; SD: Standard deviation; VIF: Variance expansion factor; CFI: Comparative fit index; RMR: Root-mean-square residual

\section{Acknowledgements}

The authors are grateful to CHARLS research team for providing the data. Roland Ligetvári has provided medical editing support, we are most thankful for his proofreading, and technical editing.

\section{Authors' contributions}

JZ contributed conception and design of the study; JZ and MJ organized the database; YX and MJ reviewed the English language and grammar. JZ performed the statistical analysis and wrote the first draft of the manuscript. All authors read and approved the final manuscript.

Funding

This study received no external funding.

\section{Availability of data and materials}

All the data can be found on the CHARLS website (http://charls.pku.edu.cn/ en). The datasets analyzed during current study are available from the corresponding author on reasonable request.

\section{Declarations}

Ethics approval and consent to participate

The ethical committees have approved the study protocol of Peking University (IRB 00001052-11014), and all the written informed consent has been provided by participants.

Consent for publication

Not applicable.

\section{Competing interests}

The authors declare that they have no competing interests.

\section{Author details}

${ }^{1}$ Department of Clinical Epidemiology, the Fourth Affiliated Hospital of China Medical University, Shenyang 110032, P. R. China. ${ }^{2}$ Department of Epidemiology, School of Public Health, China Medical University, Shenyang 110122, China. ${ }^{3}$ National Office for Maternal and Child Health Surveillance of China, Department of Obstetrics, West China Second University Hospital, Sichuan University, Chengdu 610041, P. R. China.

Received: 3 January 2021 Accepted: 21 April 2021

Published online: 30 April 2021

\section{References}

1. Cornier M-A, Dabelea D, Hernandez TL, Lindstrom RC, Steig AJ, Stob NR, et al. The metabolic syndrome. Endocr Rev. 2008;29(7):777e822.

2. Reusch JE. Current concepts in insulin resistance, type 2 diabetes mellitus, and the metabolic syndrome. Am J Cardiol. 2002;90(5A):19G-26G. https:// doi.org/10.1016/s0002-9149(02)02555-9.

3. Ormazabal V, Nair S, Elfeky O, Aguayo C, Salomon C, Zuñiga FA. Association between insulin resistance and the development of cardiovascular disease. Cardiovasc Diabetol. 2018;17(1):122.

4. Muniyappa R, Sowers JR. Role of insulin resistance in endothelial dysfunction. Rev Endocr Metab Disord. 2013;14(1):5e12.

5. Ortega-Loubon C, Fernández-Molina M, Singh G, Correa R. Obesity and its cardiovascular effects. Diabetes Metab Res Rev. 2019;35(4):e3135. https://doi. org/10.1002/dmrr.3135.

6. Sieminska E, Sobczak P, Skibinska N, Sikora J. The differential role of uric acid - the purpose or cause of cardiovascular diseases? Med Hypotheses. 2020; 142:109791. https://doi.org/10.1016/j.mehy.2020.109791.

7. Ndrepepa G. Uric acid and cardiovascular disease. Clin Chim Acta. 2018;484: 150-63. https://doi.org/10.1016/j.cca.2018.05.046.

8. Yun Lee S, Park W, Ju Suh Y, Jin Lim M, Kwon SR, Lee JH, et al. Association of Serum Uric Acid with cardiovascular disease risk scores in Koreans. Int J Environ Res Public Health. 2019;16(23):4632.

9. Maloberti A, Giannattasio C, Bombelli M, Desideri G, Cicero AFG, Muiesan $\mathrm{ML}$, et al. Working group on uric acid and cardiovascular risk of the Italian Society of Hypertension (SIIA). Hyperuricemia and risk of cardiovascular outcomes: the experience of the URRAH (uric acid right for heart health) project. High Blood Press Cardiovasc Prev. 2020;27(2):121-8. https://doi. org/10.1007/s40292-020-00368-z.

10. Rahimi-Sakak F, Maroofi M, Rahmani J, Bellissimo N, Hekmatdoos A. Serum uric acid and risk of cardiovascular mortality: a systematic review and doseresponse meta-analysis of cohort studies of over a million participants. BMC Cardiovasc Disord. 2019;19(1):218. https://doi.org/10.1186/s12872-019-1215-z.

11. Mazidi M, Katsiki N, Mikhailidis DP, Bartłomiejczyk MA, Banach M. Association of Empirical Dietary Atherogenic Indices with all-cause and cause-specific mortality in a multi-ethnic adult population of the United States. Nutrients. 2019;11(10):2323. https://doi.org/10.3390/nu11102323. 
12. Albu JB, Murphy L, Frager DH, Johnson JA, Pi-Sunyer FX. Visceral fat and race-dependent health risks in obese nondiabetic premenopausal women Diabetes. 1997:46(3):456-62. https://doi.org/10.2337/diab.46.3.456.

13. Carey DG, Jenkins AB, Campbell LV, Freund J, Chisholm DJ. Abdominal fat and insulin resistance in normal and overweight women: direct measurements reveal a strong relationship in subjects at both low and high risk of NIDDM. Diabetes. 1996;45(5):633-8. https://doi.org/10.2337/diab.45.5.633.

14. Macor C, Ruggeri A, Mazzonetto P, Federspil G, Cobelli C, Vettor R. Visceral adipose tissue impairs insulin secretion and insulin sensitivity but not energy expenditure in obesity. Metabolism. 1997;46(2):123-9. https://doi. org/10.1016/S0026-0495(97)90288-2.

15. Ponnalagu SD/O, Xinyan B, Jeyakumar HC. Is waist circumference more strongly associated with metabolic risk factors than waist-to-height ratio in Asians? Nutrition. 2019;60:30-4.

16. Rankinen T, Kim SY, Perusse L, Despres JP, Bouchard C. The prediction of abdominal visceral fat level from body composition and anthropometry: ROC analysis. Int J Obes Related Metab Disord. 1999;23(8):801-9. https://doi. org/10.1038/sj.ijo.0800929.

17. Gillum RF. The association of the ratio of waist to hip girth with blood pressure, serum cholesterol and serum uric acid in children and youths aged 6-17 years. J Chronic Dis. 1987:40(5):413-20. https://doi.org/10.1016/ 0021-9681(87)90174-3.

18. Kono S, Shinchi K, Imanishi K, Honjo S, Todoroki I. Behavioural and biological correlates of serum uric acid: a study of self-defence officials in Japan. Int J Epidemiol. 1994;23(3):517-22. https://doi.org/10.1093/ije/23.3.517.

19. Yamashita S, Matsuzawa Y, Tokunaga K, Fujioka S, Tarui S. Studies on the impaired metabolism of uric acid in obese subjects:marked reduction of renal urate excretion and its improvement by a low-calorie diet. Int J Obes. 1986;10(4):255-64.

20. Chiang KM, Tsay YC, Ng T, Yang HC, Pan WH. Is Hyperuricemia, an earlyonset metabolic disorder, causally associated with cardiovascular disease events in Han Chinese? J Clin Med. 2019;8(8):1202. https://doi.org/10.3390/ jcm8081202.

21. Bombelli M, Quartitrevano F, Tadic M, Facchetti R, Cuspidi C, Mancia G, et al. Uric acid and risk of new-onset metabolic syndrome, impaired fasting glucose and diabetes mellitus in a general Italian population: data from the Pressioni Arteriose Monitorate E Loro Associazioni study. J Hypertens. 2018; 36(7):1492-8. https://doi.org/10.1097/HJH.0000000000001721.

22. Ahmad MI, Dutta A, Anees MA, Soliman EZ. Interrelations between serum uric acid, silent myocardial infarction, and mortality in the general population. Am J Cardiol. 2019;123(6):882-8. https://doi.org/10.1016/j.amjca rd.2018.12.016.

23. Osgood K, Krakoff J, Thearle M. Serum uric acid predicts both current and future components of the metabolic syndrome. Metab Syndr Relat Disord. 2013;11(3):157-62. https://doi.org/10.1089/met.2012.0151.

24. Cicero AFG, Salvi P, D'Addato S, Rosticci M, Borghi C. Association between serum uric acid, hypertension, vascular stiffness and subclinical atherosclerosis: data from the Brisighella heart study. J Hypertens. 2014; 32(1):57-64. https://doi.org/10.1097/HJH.0b013e328365b916.

25. Mazidi M, Kengne AP, Katsiki N, Mikhailidis DP, Banach M. Inverse association between serum antioxidant levels and inflammatory markers is moderated by adiposity: a report based on a large representative population sample of American adults. Br J Nutr. 2018;120(11):1272-8. https://doi.org/10.1017/S0007114518002581.

26. von Salisch M, Zeman JL. Pathways to reciprocated friendships: a crosslagged panel study on young adolescents' anger regulation towards friends. J Youth Adolesc. 2018:47:673e87.

27. Kivimäki M, Feldt T, Vahtera J, Nurmi JE. Sense of coherence and health: evidence from two cross-lagged longitudinal samples. Soc Sci Med. 2000; 50(4):583-97. https://doi.org/10.1016/s0277-9536(99)00326-3.

28. Dehghan A, van Hoek M, Sijbrands EJ, Hofman A, Witteman JC. High serum uric acid as a novel risk factor for type 2 diabetes. Diabetes Care. 2008;31(2): 361-2. https://doi.org/10.2337/dc07-1276.

29. Rathmann W, Haastert B, Icks A, Giani G, Roseman JM. Ten-year change in serum uric acid and its relation to changes in other metabolic risk factors in young black and white adults: the CARDIA study. Eur J Epidemiol. 2007; 22(7):439-45. https://doi.org/10.1007/s10654-007-9132-3.

30. Carnethon MR, Fortmann SP, Palaniappan L, Duncan BB, Schmidt MI, Chambless LE. Risk factors for progression to incident hyperinsulinemia: the atherosclerosis risk in communities study, 1987-1998. Am J Epidemiol. 2003; 158(11):1058-67. https://doi.org/10.1093/aje/kwg260.
31. Zhu Y, Hu Y, Huang T, Zhang Y, Li Z, Luo C, et al. High uric acid directly inhibits insulin signalling and induces insulin resistance. Biochem Biophys Res Commun. 2014;447(4):707-14. https://doi.org/10.1016/j.bbrc.2014.04.080.

32. Viazzi F, Garneri D, Leoncini G, Gonnella A, Muiesan ML, Ambrosioni E, et al. Serum uric acid and its relationship with metabolic syndrome and cardiovascular risk profifile in patients with hypertension: insights from the I-DEMAND study. Nutr Metabol Cardiovasc Dis. 2014;24(8):921e7.

33. Tseng WC, Chen YT, Ou SM, Shih CJ, Tarng DC, Taiwan Geriatric Kidney Disease (TGKD) Research Group. U-Shaped Association Between Serum Uric Acid Levels With Cardiovascular and All-Cause Mortality in the Elderly: The Role of Malnourishment. J Am Heart Assoc. 2018:7(4):e007523.

34. Hu L, Hu G, Xu BP, Zhu L, Zhou W, Wang T, et al. U-Shaped Association of Serum Uric Acid With All-Cause and Cause-Specific Mortality in US Adults: A Cohort Study. J Clin Endocrinol Metab. 2020;105(1):dgz068.

35. Simental-Mendía LE, Rodríguez-Moran M, Guerrero-Romero F. The product of fasting glucose and triglycerides as surrogate for identifying insulin resistance in apparently healthy subjects. Metab Syndrome Relat Disord. 2008;6(4):299e304

36. Martínez-Larrad MT, Lorenzo C, González-Villalpando C, Gabriel R, Haffner SM, Serrano-Ríos M. Associations between surrogate measures of insulin resistance and waist circumference, cardiovascular risk and the metabolic syndrome across Hispanic and non-Hispanic white populations. Diabet Med. 2012;29(11):1390-4. https://doi.org/10.1111/j.1464-5491.2012.03723.x.

37. Björnson $E$, Adiels M, Taskinen MR, Borén J. Kinetics of plasma triglycerides in abdominal obesity. Curr Opin Lipidol. 2017;28(1):11-8. https://doi.org/10.1 097/MOL.0000000000000375

38. Carpentier AC. Hypertriglyceridemia associated with abdominal obesity: getting contributing factors into perspective. Arterioscler Thromb Vasc Biol. 2015;35(10):2076-8. https://doi.org/10.1161/ATVBAHA.115.306412.

39. Wang Y, Rimm EB, Stampfer MJ, Willett WC, Hu FB. Comparison of abdominal adiposity and overall obesity in predicting risk of type 2 diabetes among men. Am J Clin Nutr. 2005;81(3):555-63. https://doi.org/10.1093/a $\mathrm{jcn} / 81.3 .555$.

40. Niskanen L, Laaksonen DE, Lindström J, et al. Serum uric acid as a harbinger of metabolic outcome in subjects with impaired glucose tolerance. Diabetes Care. 2006;29(3):709-11. https://doi.org/10.2337/diacare.29.03.06. dc05-1465.

\section{Publisher's Note}

Springer Nature remains neutral with regard to jurisdictional claims in published maps and institutional affiliations.

Ready to submit your research? Choose BMC and benefit from:

- fast, convenient online submission

- thorough peer review by experienced researchers in your field

- rapid publication on acceptance

- support for research data, including large and complex data types

- gold Open Access which fosters wider collaboration and increased citations

- maximum visibility for your research: over $100 \mathrm{M}$ website views per year

At $\mathrm{BMC}$, research is always in progress.

Learn more biomedcentral.com/submission 